Kendig, Catherine (2014). "Synthetic Biology and Biofuels." In Paul B. Thompson and David M. Kaplan, eds., Encyclopedia of Food and Agricultural Ethics. Dordrecht: Springer.

\title{
Synthetic Biology and Biofuels
}

Catherine Kendig, Department of Philosophy and Religion, Missouri Western State University, Saint Joseph, Missouri, U.S.A., ckendig@missouriwestern.edu

Synonyms

Genetic engineering, biological engineering, genetically modified, GM, biogas, green energy, energy, crop fuel, biodiesel, biomass, renewable, renewable energy

Introduction

Synthetic biology is a field of research that concentrates on the design, construction, and modification of new biomolecular parts and metabolic pathways using engineering techniques and computational models. By employing knowledge of operational pathways from engineering and mathematics such as circuits, oscillators, and digital logic gates, it uses these to understand, model, rewire, and reprogram biological networks and modules. Standard biological parts with known functions are catalogued in a number of registries (e.g. Massachusetts Institute of Technology Registry of Standard Biological Parts). Biological parts can then be selected from the catalogue and assembled in a variety of combinations to construct a system or pathway in a microbe. Through the innovative re-engineering of biological circuits and the optimization of certain metabolic pathways, biological modules can be designed to reprogram organisms to produce products or behaviors.

Synthetic biology is what is known as a "platform technology". That is, it generates highly transferrable theoretical models, engineering principles, and know-how that can be applied to create potential products in a wide variety of industries. Proponents suggest that applications of synthetic biology may be able to provide scientific and engineered solutions to a multitude of worldwide problems from health to energy. Synthetic biology research has already been successful in constructing microbial products which promise to offer cheaper pharmaceuticals such as the antimalarial synthetic drug artemisinin, engineered microbes capable of cleaning up oil spills, and the engineering of biosensors that can detect the presence of high concentrations of arsenic in drinking water.

One of the potential benefits of synthetic biology research is in its application to biofuel production. It is this application which is the focus of this entry. The term "biofuel" has referred generally to all liquid fuels that are sourced from plant or plant byproducts and are used for energy necessary for transportation vehicles (Thompson 2012). Biofuels that are produced using synthetic biological techniques re-engineer microbes into biofuel factories are a subset of these.

Entry roadmap 
This entry begins with a short historical background that focuses on initial ethical support and justification for synthetic biofuel research, the impact of this research on public discussion of synthetic biology, and the distinction between it and genetic engineering. The distinction between first and second generation biofuels is introduced. This is followed by a survey of current research innovations using various microbial factories, including: bacteria, yeast, and oil (oleaginous) algae.

Ethical considerations associated with synthetic biology research in general and its application to biofuel production in particular will be reviewed. General responses by opponents of all forms of synthetic biology include the claim that this type of technology aims to "play God" and that the unnaturalness of it intervenes in the natural world in ways that are unethical and should therefore be avoided. This justification has been used to attempt to restrict or stop new approaches to biofuel technology that aim to control and co-opt natural selection in order to produce a stable product.

Proponents of this synthetic re-engineering suggest that these ethical concerns are unfounded. Synthetic biology merely extends the mechanisms by which artificial selection can be controlled and modified beyond traditional approaches to selective breeding.

Ethical considerations that apply specifically to synthetic biofuel research and technology include issues in the design, construction, implementation, marketable production, and assessment of synthetic biofuel production when compared to food crop biomass-based biofuels. Motivations for synthetic applications that focus on the growing concerns over the high cost of production of crop biomass produced biofuels and the subsequent food shortages that followed, widely framed in terms of the food versus fuel debate will be discussed. In addition to these, the ethical issues surrounding the potential impact on human health and the environment consequences of intentional and accidental release of synthetic products of biofuel research will also be covered.

Ethical discussion surrounding synthetic biology and biofuels is, like the research and technology itself, still emerging. An outline of the current efforts of commissions and consortia set up in the United States and the United Kingdom that have promoted the scientifically informed open exchange of ideas between scientists and the public on ethical issues relating to synthetic biology research and application are discussed.

Historical background

Synthetic biofuel production seeks to provide less expensive, cleaner, and greener sources of energy than currently used traditional fossil fuels. Perhaps because of this, it has been one of the most publically accepted and perhaps most promising applications of synthetic biology. In a field of research where descriptions of the products of synthetic biology are frequently reported as "designer organisms", "Frankencell", or the result of "playing God", discussion of synthetic biofuels seems less controversial. Its potential benefits are often weighed up against its potential costs: "synthetic biology 
poses a conundrum because of its double-edged ability to both wreak biological havoc and perhaps wean civilization from dirty $20^{\text {th }}$ century technologies and petroleum-based fuels" (Weiss 2007).

Although sometimes referred to as genetic engineering, synthetic biology differs from genetic engineering in terms of scale, techniques of manipulation, and application. Genetic engineering focuses on the alteration or manipulation of a few characteristics of an organism that results in transgenic hybrids or genetic chimeras that possess genes inserted from other organisms. Whereas, synthetic biology seeks to reconfigure, design, and construct new pathways, whole processes, or novel systems for the purpose of achieving some desired biosynthetic activity or phenotype (Alper and Stephanopoulos 2009).

First generation biofuel production strategies

Current research aims at producing a cleaner biofuel alternative to those that are currently agriculturally produced using food crops. These crop-based biofuels, often referred to as first generation biofuels, rely on agricultural crops as biomass to produce sugar or starch from corn, wheat, or barley and convert this to ethanol through fermentation and distillation processes, or rely on oilseed crops to produce triacylglycerols that are then chemically converted to biodiesel.

One of the most heated recent ethical discussions focusing on the use of first generation biofuel production has been the so-called food vs. fuel debates. These have typically centered on discussions about the appropriate use of agricultural land-should agricultural land be used for generating fuel instead of food? The decisions of some farmers to plant food crops such as maize for the purpose of harvesting the biomass to sell to biofuel producers has been controversially linked with an increase in food cost and food shortages.

In addition to the fuel versus food debates, discussion has also focused on the production costs themselves and their impact on the environment. First generation agricultural crop based biofuel production demands arable land use, water use in irrigation, and provides a fuel source that may not be as cost-effective or renewable as the potential second generation biofuels which do not require either arable land, irrigation, or displace food crops (Börjesson and Mattiasson 2008).

Second generation biofuel research strategies

Instead of relying on food crops as the source of fuel, synthetic engineered alternatives, or second generation biofuels, rely on using the redesigned microbial cell as the source of biofuel production. Synthetic biological engineering is sometimes referred to as white biotechnology due to its focus on renewable energy sources and the reduction of negative environmental effects and the potential biodegradability of its engineered products.

In providing more sustainable avenues of biofuel production, these second generation synthetic biofuel alternatives present a way to circumvent the controversial fuel versus food debates that have been widely discussed in the various news media and in local and global ethical discussions. 
These and other debates have led private and public support of synthetic biology research into carbonneutral synthetic biofuel alternatives that may provide solutions to not only the energy crisis but also provide a way to circumvent the continued degradation of the environment through the burning of fossil fuels and greenhouse gas emissions (Martin et al. 2003).

Microbial factories of biofuel production: prospects and problems

Synthetic biology builds on past successes and failures-what works in terms of both modules that are highly interchangeable as well as methods and pathways are reused in new projects. Earlier research on another application of synthetic biology research, the production of synthetic artemisinin, provided guidance on how microbes can be used and their tolerance of different types of chemical products. Synthetic artemisinin is perhaps one of the most widely discussed success stories of synthetic biology research. Artemisinin is an anti-malarial drug which is now sold commercially by Amyris Biotechnologies which now uses similar technology in the search for a scalable renewable biofuel (Amyris Biotechnologies 2013).

Development of second generation biofuel requires the use of a microbial factory organism such as the yeast, Saccharmyces cervisiae, the bacterium, Escherichia coli, or the single-celled green alga, Chlamydomonas reinhardtii, which is then redesigned to produce certain products. To do this, synthetic biology researchers use parts with known functions from a variety of organisms. A catalogue of these parts is used to choose which components can be put together in the cell to build new pathways and as a result produce new chemical products.

One of the initially most promising organisms to construct a potential biofuel factory was the yeast. $S$. cervisiae. This is widely used to produce ethanol from sugar in the brewing of beer. As it is also an organism frequently used in genetics and synthetic biology research, its metabolic pathways and functions are well characterized. Its ability to produce ethanol made it appear to be a good beta test for a new biofuel factory. E.coli is another common organism for use in synthetic biological research in virtue of its proven ability to accept genetic modification with the introduction of foreign genes, the tendency to maintain hybrid networks, and the production of a variety of products (Martin et al. 2003)., For instance, Fuzhong Zhang, James Carothers, and Jay Keasling, have successfully designed a strain of e.coli that implements a biosensor for a metabolite that plays a role in the production of biofuel products using glucose (Zhang, Carothers, and Keasling 2012). By adding the biosensor to the engineered pathway, their new strain of $e$. coli is capable of trebling the amount of fuel produced (Zhang, Carothers, and Keasling 2012).

Relying on the knowledge of these and other microbial pathways and modules means that these parts can be both modified and transferred into other organisms to re-engineer it to produce a higher ethanol yield or to produce ethanol by consuming a different sugar (such as hexose or xylose) rather than what it would normally utilize (e.g. glucose). 
The current research trend in publically and privately funded projects is to investigate the potential use of various species of algae for biofuel production. Algae produce lipids (oil) as a byproduct of the process of photosynthesis. The hope is that once the means of harnessing this store of energy is found, algal biofuels may provide an inexpensive alternative source of fuel that can be produced with little more than sunlight, carbon dioxide, and a small amount of water. While advances in synthetic biology research and the understanding of algal alternatives increases, the scaling up of these fuels requires significant further research resolving the problems of system optimization and photosynthetic efficiency as well as solving ways of producing these synthetic biofuels in quantities suitable for commercial use (Georgianna and Mayfield 2012).

A number of other possible organisms have also been considered as particularly suitable for research into the production of synthetic biofuels. Cyanobacteria are another that initially appears promising. Cyanobacteria, like Synechocystic sp. PCC 6803, can provide a highly efficient organic system for producing biofuels as they can convert solar energy and carbon dioxide into biofuel molecules (Wang, Liu, \& Lu 2013). Cyanobacteria are particularly good candidates because they possess naturally occurring biosynthetic pathways that produce alkane (a key component of gasoline, diesel, and jet fuel). At present, research into the use of cyanobacteria for synthetic biofuel production is still in the very early stages and well behind that of algae research. However, research focused on reconfiguring these to create an organism that produces alka(e)ne at a rate that is double that of the wild type has been shown to be possible. Synechocystis mutants have been constructed that overexpress alkane biosynthetic genes. This research demonstrates proof-of-concept for the potential use of cyanobacteria for biofuel production. If their photosynthetic pathways were re-engineered, cyanobacteria may be able to produce alka(e)nes at a highly efficient rate (Wang, Liu, \& Lu 2013).

Synthetic biofuel production relying on either cyanobacteria or algae may provide a possible alternative to fossil fuels. The ethical consequences on the environment have primarily focused on how this new technology would (by reducing the reliance on the burning of fossil fuels and anthropogenic climate change), contribute to a cleaner, greener planet. Other ethical impacts concerning the potential negative effects (to not only the environment but also on human health) have been raised by Friends of the Earth (2013). Exposure to synthetic biological materials by lab technicians raises a particular set of concerns that centers on both the kinds of products produced by synthetic biology and their potential risks. The potential for accidental ingestion (e.g. breathing aerosol versions of synthetically produced algae biofuel), genetic transfer through viral, or nanomaterial by lab technicians, or the potential escape into the environment of such products have been discussed. These are a concern because of the projectible negative consequences, but more so for the unexpected and potentially catastrophic impact on human health (e.g. that ingestion of synthetic materials may lead to disastrous unintentional modifications of the human genome, epigenome, and microbiome) (Hoffman, Hanson \& Thomas 2013).

Production problems and solutions: Tricking biological systems to redirect the process of evolution 
Although promising, the re-engineering of biological pathways to produce high-yield microbial biofuel factories has encountered some formidable obstacles in virtue of their biological basis. Synthetically engineered organisms, circuits, parts, and systems, like their organic counterparts, have the capacity to adapt to new environments and to evolve over generations. Because these engineered synthetic microbes are biological systems that continue to have the propensity to evolve and mutate, understanding how to design an organism that has predictable behavior is difficult. Their functioning is designed according to the interests of the particular application (e.g. biofuel production) within the lab.

If these built circuits prove cumbersome for the organism once it is in the natural environment, they will be replaced by more evolutionarily suitable pathways. Engineered circuits created in them that do not provide a benefit to the organism may be disposed of in subsequent generations. That is, if producing the high-yield byproducts that they are designed to produce does not provide the organism with an evolutionary advantage or increased fecundity, it is likely that this pathway may be lost in subsequent generations. Once a mutation occurs in a later generation that removes part of the biologically taxing pathway responsible for the high-yield production, these mutated organisms may gain an evolutionary advantage over those with the engineered pathway (Kendig 2012, 2013). Over generations, this would eventually lead to a population that would lack the engineered pathway and one where the mutation would be common. As a consequence of this natural selection, the resulting population would produce a lower biofuel yield (Kendig 2012, 2013).

To solve this problem, researchers are currently seeking ways to trick the biological system and redirect the process of evolution for the purposes of producing a higher yield product than the organism would produce (Jia, Zhang, and Li 2010). Finessing the organism's circuitry so that it is stable in a variety of environmental conditions and continues to produce high yield products is of paramount importance. Ethical and environmentally responsible release of an organism that is fully characterized in the controlled context and known parameters of the lab to a new and changing context of the environment requires prior knowledge not only of the organism or circuit's design and functionality, but also its potential mutability and evolvability in an uncontrolled environment.

Ethical concerns over the unpredictability of potential and irreversible impacts on ecosystems

Evolvability and the co-opting of mutability is not just a production problem, it also opens up a number of ethical considerations. These questions make up a more nuanced set of issues that relate specifically to synthetic biofuel production. Rather than the more often broadly referred to ethical concerns mentioned when discussants claim that this kind of technology should not be advance because it involves scientists "playing God", these concerns are directly informed through an understanding of the specifics of biofuel research and production. Much of the worry with regard to co-opting of evolvability are the downstream effects or unforeseen consequences of "meddling" with nature.

A frequently used rebuttal to worries that synthetic biologists are "playing God" is the suggestion that farmers have been crossing-breeding livestock , companion breeds of animals and food crops for a long time and that synbiology is just a technological extension of this. As such, proponents, conclude, ethical 
concerns over biologists overreaching in the creation of these synthetically re-engineered organisms are thought to be unfounded. The morality of these kinds of synthetic interventions has already been treated to a long and relatively unproblematic beta test in the thousands of years of artificial breeding. Opponents counter that the new technology is dissimilar enough to cause ethical concerns unrelated to that history.

Coalitions such as those that make up the undersigners of the Principles of oversight for synthetic biology (2013): a document of the International Center for Technology Assessment, ETC Group and Friends of the Earth, have also been instrumental in the initial ethical discussion surrounding synthetic biology research and concerns about the unpredictability of risks and worries over their possible impacts. This coalition includes civil, social, labor, as well as religious groups concerned with multiple potential impacts that include those economic and environmental.

It raises concerns about the rapidity of scientific research and innovation in synthetic biology without appropriate regulation or consideration of potential risks. They advocate the explicit use of the Precautionary Principle, specific regulations on synthetic biology, an assessment of harm to public health and environment, increased access to synthetic biology research and active participation of public fora, liability and accountability of manufacturers of synthetic biology technology, and an increased effort to protect the economic interests of environmentally vulnerable groups and countries (Hoffman, Hanson, \& Thomas 2013).

The undersigners suggest that in order to preclude potential damage that could result from the products of synthetic biology research, "Governmental bodies, international organizations and relevant parties must immediately implement strong precautionary and comprehensive oversight mechanisms enacting, incorporating and internalizing these basic principles. Until that time, there must be a moratorium on the release and commercial use of synthetic organisms and their products to prevent direct or indirect harm to people and the environment"(Hoffman, Hanson, \& Thomas 2013). This moratorium is justified by the coalition because of the potential for long-term harm to the environment. The document suggests that the risks to niche degradation may be long-lasting. For instance, synthetic organisms could be the new super-invasive species crowding out other native species within a particular ecological niche. If synthetic organism parts are highly modular, their genes may lead to the contamination of other species by virtue of their high level of transferability within the environment through horizontal gene transfer.

The shape of ethical discussion so far

Ethical discussion surrounding synthetic biofuels has come in two sorts so far. The first suggests that the ethical issues that synthetic biology addresses are the same as other emerging technologies, and fundamentally contiguous with those that have been and continue to be discussed. These are the traditional ethical questions of moral behaviour, rights and responsibilities, and questions of moral agency. Whilst the other suggests that emerging technologies each present fundamentally new sets of ethical issues. 
The latter suggests that the resolution to these issues requires consideration not only of the ethical reasoning supplied to us by reading Kant, Aristotle, Bentham, Mill, Anscombe, or Hobbes' approaches to the questions of how should we act, or questions concerning what kinds of characteristics are good for someone to have. But they also require engagement with the specific scientific research and technology itself in order to fully inform ethical reasoning. Both are required to answer questions such as: What are the limits of human intervention within the natural world? Should we intervene and co-opt the selective processes of evolution and bend these to our will? How should we behave as researchers?, what responsibilities do we have for society, the environment, and for the unintended effects of the new technology created? Who owns the products of these types of research or the rights to use the technology once patented?

Insofar as synthetic biology is a relatively new form of scientific research and the applications to biofuel in their early stages, ethical discussion surrounding these is still in the investigative stages of development relying on panoply of reference points to gain traction on new ethical questions.

The ethical discussions of synthetic biology research in general and the application to produce biofuels in particular have progressed along very different routes to that of the discussions surrounding genetically modified foods. The most striking difference has come in the public perception of this new technology and the encouraged exchange between research bodies and the public in open forums. Commissions and research units such as the aforementioned U.S. Presidential Commission for the Study of Bioethical Issues, the Hastings Center Ethical Issues in Synthetic Biology project (launched in 2009 and funded by the Alfred P. Sloan Foundation), and the SYNBIOSAFE consortium, (set up in 2007 and funded by the European Commission), have promoted scientifically-informed ethical discussions that bring together key researchers, policy makers, academics, and the public with the goal that through dialogue there can be increased understanding and appropriate regulation of this new biotechnology in a way that is responsive to the interests of scientific innovation and public concern.

In doing so, these cross-disciplinary bodies aim to focus less on the speculative ethical debates of the potential problematic products or misuses of synthetic biology research. Instead, their foci are on current research problems and issues with practical applications in the not-too-distant future. Open debates which inform the public about current research, objectives, and technological applications rather than spurious conjecturing based on worries derived from science fiction and hypothetical slippery slope arguments have been the goal of these commissions and consortia.

Towards scientifically-informed ethical discussion

The promise of a cheaper, greener alternative to fossil fuels is an attractive application of synthetic biology research. But with the capability to construct organisms for this and other applications comes a veritable flotilla of ethical considerations. Including those already discussed in the foregoing, these concern each stage of development, from research and design, to the production, use, regulation, impacts on the environment, modes of release and dissemination, public perception, and marketing. 
In response to the J. Craig Venter Institute's 2010 announcement that they had created "synthetic life" by digitally crafting DNA and inserting it into a living bacterium to produce a new self-replicating bacterium, Mycoplasma mycoides JCVI-syn1.0, the United States Presidential Commission for the Study of Bioethical Issues produced the report, New Directions: The Ethics of Synthetic Biology and Emerging Technologies. This report provided guidance and consideration of policies, governance, and practices that would enable synthetic biology research and applications of it to be pursued in an "ethically responsible manner" but did not endorse further federal regulations on synthetic biology research (Presidential Commission for the Study of Bioethical Issues 2010). In doing so, The Presidential Commission followed the recommendations already laid out by the Engineering and Physical Sciences Research Council, the Biotechnology and Biological Sciences Research Council, the Economic and Social Research Council and the Arts and Humanities Research Council of the United Kingdom (Anderson et al. 2012).

In the New directions report, the Presidential Commission set out five principles to guide discussion of the ethical and social impacts of synthetic biology research and technological applications. The report's recommendations are framed in terms of these five principles. These are: intellectual freedom, democratic deliberation, responsible stewardship, and considerations of justice and fairness. With intellectual freedom, responsibility for the implications of synthetic biology research and technological applications is put largely in the hands of the researchers to self-regulate rather than promoting a topdown approach for regulating practice. The U.S. and U. K. initiatives stress prudence and responsiveness with regard to the emerging area of research still in its infancy.

Crucial issues mentioned within the U. S. report focus on responsible stewardship to the world and its occupants, including considerations of non-human animals, plants, and future generations, as well as the environment as a whole. Consideration of these groups within the context of a discussion of obligations is not new. Although not cited, the report's main ethical foci seem to rely significantly on philosophical ideas similar to those laid out in 1990 by Edith Brown Weiss in her seminal article, "Our Rights and Obligations to Future Generations for the Environment". To summarize, Weiss suggests that the rights of each generation are to receive the planet in no worse condition than did the generation that came before it. This would mean that each generation should inherit comparable natural diversity and have similar access to the environment and its resources as did the previous generation. She suggests that rights and obligations do not arise de novo, but instead derive from an intergenerational relationship that each generation shares with those in the one preceding it as well as those in the subsequent generation yet to come (Weiss 1990).

\section{Summary}

Scarcity of resources and the unsustainability of the continued use of fossil fuels drive the synthetic biological engineering of biofuels. The initial sources of biofuels based on collecting and fermenting the biomass of food crops (such as corn), proved highly controversial. The growth of corn ethanol producers and the increase in food shortage attributed to the subsequent worldwide backlash and contributed to much critical discussion. These first generation biofuel discussions concentrated on the ethical impacts 
of agricultural land use for biofuel crops and limited water supplies previously used to produce food that were now being used to produce fuel instead.

Despite the problems with first generation biofuels, investment in second generation synthetic biofuels by private companies as well as government support of research (especially in the U.S.) has been significant (Tyner, Dooley, and Viteri 2011). If the demand and pursuit of liquid transportation fuels continues, synthetically re-engineered alternatives which provide functional equivalents to fossil fuels may be the greenest option as they may offer a more renewable avenue to the production of fuel.

Although synthetic biofuel production shows much promise as an alternative energy source that does not require the use of non-sustainable feedstocks or expensive carbon sources, its ability to replace fossil fuel consumption rests on developing the technology to produce it economically whilst reducing any negative environmental impacts. Despite their overuse, contribution to anthropogenic climate change, and the source of frequent international disputes, the replacement of fossil fuels will still rely overwhelmingly on economic production of an efficient, high yield alternative source of energy (Georgianna and Mayfield 2012).

As synthetic biology is still a new field of research and only some of the potential applications are just being realized, open discussion with scientists, policy makers, and the public may provide the best prospects according to many commissions and consortia in the U.S. and U.K. Discussion of the actual scientific research and its accurate dissemination to media and the public would allow productive and democratic exchanges of a well-informed public and a responsive scientific community collaborating to evaluate the direction of new research. The goal is for these discussions to stimulate active enlightened deliberation directed towards navigating the best route(s) for ensuring the pursuit of this research, critical evaluation of its potential positive and negative impacts.

Cross-references

Biofuels: Ethical Aspects, Land Acquisition for Food and Fuel, Energy, Climate Change and Food, Synthetic Biology and Food, Agriculture and Environmentalism, Hybridity in Agriculture, Water Use in Agriculture

References

Alper, H. \& Stephanopoulos, G. (2009). Engineering for biofuels: exploiting innate microbial capacity or importing biosynthetic potential? Nature Reviews Microbiology, 7, 715-723.

Amyris Biotechnologies (2013). Company website at: http://www.amyrisbiotech.com/Innovation/155/BreakthroughScience Accessed 3/15/2013.

Anderson, J., Strelkowa, N., Stan, G-B, Douglas, T., Savulescu,J., Barahona, M., \& Papachristodoulou, A. (2012). Engineering and ethical perspectives in synthetic biology. EMBO reports, 13(7), 584-590.

Börjesson, P. \& Mattiasson, B. (2008). Biogas as a resource-efficient vehicle fuel. Trends in Biotechnology, 26, 7-13.

Georgianna, R. \& Mayfield, S. (2012). Exploiting diversity and synthetic biology for the production of algal biofuels. Nature, 488, 329-335. 
Hoffman, E., Hanson, J., \& Thomas, J. (2013). The Principles for the Oversight of Synthetic Biology. Declaration drafted by the Friends of the Earth U.S., International Center for Technology Assessment, ETC Group. http://libcloud.s3.amazonaws.com/93/ae/9/2287/2/Principles_for_the_oversight_of_synthetic _biology.pdf. Accessed 11-09-2013.

Jia, K., Zhang, \& Y. Li, Y. (2010). Systematic engineering of microorganisms to improve alcohol tolerance. Engineering in Life Sciences, 10(5), 422-429.

Kendig, C. (2012). Philosophical investigations of diverse methods in synthetic biology. Presentation at the Genome Consortium for Active Teaching (GCAT) Synthetic Biology workshop. Ashburn, VA: Howard Hughes Medical Institute Janelia Farm Research Campus. Accessed 22 June 2012. http://www.nsf.gov/awardsearch/showAward.do?AwardNumber=1127271

Kendig, C. (2013) How synthetic biology reconfigures biological understanding and ethical categories. Genome Consortium for Active Teaching (GCAT) Synthetic Biology workshop. Ashburn, VA: Howard Hughes Medical Institute, Janelia Farm Research Campus. Accessed 28 June 2013. http://www.nsf.gov/awardsearch/showAward.do?AwardNumber=1127271.

Martin, V., Pitera, D., Withers, S., Newman, J., \& Keasling, J. (2003). Engineering a mevalonate pathway in Escherichia coli for production of terpenoids. Nature Biotechnology, 21, 796-802.

Presidential Commission for the Study of Bioethical Issues (2010). New directions: The ethics of synthetic biology and emerging technologies. Washington, D.C.: Government Printing Office.

Preston, C. (2008). Synthetic biology: drawing a line in Darwin's sand. Environmental Values, 17, 23-40.

Thompson, P. 2012. The agricultural ethics of biofuels: climate ethics and mitigation arguments. Poiesis and Praxis, 8, 169-189.

Tyner, W., Dooley, F., \& Viteri, D. (2011). Alternative pathways for fulfilling the RFS mandate. American Journal of Agricultural Economics, 93, 465-472.

Wang, W., Liu, X.,\& Lu, X. (2013). Engineering cyanobacteria to improve photosynthetic production of alka(e)nes. Biotechnology for Biofuels 6:69. Accessed 1/11/2013 http://www.biotechnologyforbiofuels.com/content/6/1/69

Weiss, E. (1990). Our rights and obligations to future generations for the environment. The American Journal of International Law, 84(1), 198-207.

Weiss, R. (2007). Synthetic DNA on the brink of yielding new life forms. The Washington Post. December 17.

Zhang, F., Carothers, J., \& Keasling, J. (2012). Design of a dynamic sensor-regulator system for production of chemicals and fuels derived from fatty acids. Nature Biotechnology, 30 (4),354360. 